\title{
The ventromedial prefrontal cortex in a model of traumatic stress: fear inhibition or contextual processing?
}

\author{
Zachary T. Pennington, ${ }^{1}$ Austin S. Anderson, ${ }^{1}$ and Michael S. Fanselow ${ }^{1,2}$ \\ ${ }^{1}$ Department of Psychology, UCLA, Los Angeles, California 90095, USA; ${ }^{2}$ Brain Research Institute, UCLA, Los Angeles, \\ California 90095, USA
}

\begin{abstract}
The ventromedial prefrontal cortex (vmPFC) has consistently appeared altered in post-traumatic stress disorder (PTSD). Although the vmPFC is thought to support the extinction of learned fear responses, several findings support a broader role for this structure in the regulation of fear. To further characterize the relationship between vmPFC dysfunction and responses to traumatic stress, we examined the effects of pretraining vmPFC lesions on trauma reactivity and enhanced fear learning in a rodent model of PTSD. In Experiment 1, lesions did not produce differences in shock reactivity during an acute traumatic episode, nor did they alter the strength of the traumatic memory. However, when lesioned animals were subsequently given a single mild aversive stimulus in a novel context, they showed a blunting of the enhanced fear response to this context seen in traumatized animals. In order to address this counterintuitive finding, Experiment 2 assessed whether lesions also attenuated fear responses to discrete tone cues. Enhanced fear for discrete cues following trauma was preserved in lesioned animals, indicating that the deficit observed in Experiment 1 is limited to contextual stimuli. These findings further support the notion that the vmPFC contributes to the regulation of fear through its influence on context learning and contrasts the prevailing view that the vmPFC directly inhibits fear.
\end{abstract}

Of the purported biomarkers for post-traumatic stress disorder (PTSD), reductions in the size and activity of the ventromedial prefrontal cortex (vmPFC) are among the most consistent (Rauch et al. 2003; Milad et al. 2005, 2009; Richert et al. 2006; Etkin and Wager 2007; Kasai et al. 2008; Koenigs and Grafman 2009; Kühn and Gallinat 2013). Anatomically, the vmPFC is richly interconnected with other brain regions known to support the acquisition and expression of fear-including the hypothalamus, periaqueductal gray, ventral hippocampus, and basolateral amygdala (Hurley et al. 1991; Vertes 2004) — placing it in a prime position to regulate fear behaviors. Research to date has predominantly focused on the contributions of the vmPFC to the extinction of learned fear responses, as inhibition of the vmPFC is able retard tone fear extinction, and activity within the human vmPFC has been linked to fear extinction (Milad et al. 2005, 2009; Quirk and Beer 2006; Sierra-Mercado et al. 2006, 2011; Laurent and Westbrook 2009; Do-Monte et al. 2015). Such findings provide a potential bridge between biomarker and disease process.

Nevertheless, several findings are at odds with the simple hypothesis that the vmPFC supports extinction learning. First, activity of the vmPFC is also altered during the acquisition and expression of fear in humans (Phelps et al. 2004; Shvil et al. 2014) and patients with PTSD display altered activity in the vmPFC during fear acquisition/expression (Britton et al. 2005; Etkin and Wager 2007). Second, inactivation of the vmPFC has been shown to augment levels of fear in response to the presentation of a tone previously paired with shock, and activation of the vmPFC is able to decrease freezing in a similar manner (Sierra-Mercado et al. 2006, 2011; Do-Monte et al. 2015). Given that these changes are present on the very first exposure following conditioning, these findings

Corresponding author: fanselow@psych.ucla.edu

Article is online at http://www.learnmem.org/cgi/doi/10.1101/lm.046110.117. are inconsistent with an extinction interpretation. Reports have also shown that manipulations of the vmPFC are able to alter a range of unconditioned anxious behaviors (Adhikari et al. 2015). These findings more firmly suggest that the vmPFC regulates the expression of fear rather than extinction learning, per se. Alternatively, other reports suggest that vmPFC modulates fear based upon the extent to which it is controllable (Amat et al. 2005), further complicating the role of the vmPFC in the regulation of fear.

In order to better understand the contribution of the vmPFC to behavior following trauma, we assessed the impact of pretraining excitotoxic lesions on stress enhanced fear learning (SEFL), a model of PTSD that our laboratory has developed. In this model, animals that receive a severe acute stressor (the "trauma") display an enhancement of subsequent fear learning about mild stressors, similar to that seen in PTSD (Rau et al. 2005; Rau and Fanselow 2009). This model has the benefit of capturing an array of relevant phenotypes: trauma reactivity, associative recall of the traumatic event, fear generalization, and enhancement of fear learning.

Counter to the anticipated prediction that lesions of the vmPFC would enhance stress responses in the SEFL model, in line with evidence that the vmPFC supports fear inhibition, in Experiment 1 we found that pretraining lesions of the vmPFC reduced SEFL without altering any other measured phenotype. To explore this counterintuitive result, in Experiment 2 we used a modified version of the SEFL procedure to assess whether the reduction in enhanced fear learning was specific to contextual

\footnotetext{
(C) 2017 Pennington et al. This article is distributed exclusively by Cold Spring Harbor Laboratory Press for the first 12 months after the full-issue publication date (see http://learnmem.cshlp.org/site/misc/terms.xhtml). After 12 months, it is available under a Creative Commons License (AttributionNonCommercial 4.0 International), as described at http://creativecommons. org/licenses/by-nc/4.0/.
} 
stimuli, as prior research has implicated the vmPFC in contextual processing (Kesner et al. 1996; Ragozzino et al. 1998; Zelikowsky et al. 2013b).

\section{Results}

\section{Analysis}

All data were analyzed using SPSS (v23). When data fit assumptions of the general linear model, ANOVAs were used to assess omnibus differences followed by post hoc $t$-tests controlling for multiple comparisons. For cases in which low levels of freezing were anticipated in nonshocked animals and high levels of freezing were anticipated in shocked animals, data was square root transformed to correct for heterogeneity of variance (figures depict nontransformed data). If square root transformation was not sufficient to correct for heterogeneity across shock conditions, nonparametric tests (Mann Whitney $U$ ) on nontransformed data were first used to confirm differences among shocked and nonshocked animals, and the effect of lesion was subsequently assessed using traditional parametric methods for each level of the shock variable separately. Notably, ANOVA on such data rendered the same outcomes.

In Experiment $1(n=32)$, one animal in the nonlesion group was excluded for failing to freeze at all in the trauma context, indicative of an extreme outlier. Additionally, two animals were excluded from the analysis of Day 1 in Experiment 1 due to a computer error occurring when writing the data to disk at the end of the session. In Experiment 2, only the first 10 trials of Day 1 were included in statistical analysis due to a computer error that resulted in the loss of the last five trials of data for a subset of animals.

\section{Experiment 1: impact of vmPFC lesions on enhanced contextual fear learning following trauma}

An experimental timeline for Experiment 1 can be seen in Figure $1 \mathrm{~A}$.

\section{Histology}

Post-experiment immunohistochemical staining of the neuronspecific marker NeuN revealed a nearly complete loss of neurons in the infralimbic prefrontal cortex. Additionally, there was loss of cells, although to a lesser extent, in ventral portions of the prelimbic prefrontal cortex, and in some animals, slight loss in the medial orbital prefrontal cortex, just anterior to the infralimbic cortex. The extent of the lesion and a representative coronal section at the central focus of the lesion can be seen in Figure 1C,D. Two animals that had been infused with NMDA were excluded for not displaying evidence of a lesion.

\section{Day 1-Trauma}

Two weeks after the induction of excitotoxic lesions of the vmPFC, animals were placed in the trauma context, Context $\mathrm{A}$, and given either 0 or $15,1 \mathrm{sec}, 1 \mathrm{~mA}$, unsignaled shocks distributed over a 90-min period. There was no effect of lesion on post-shock freezing during this session (Lesion: $F_{(1,11)}=0.93, P=0.36$; Lesion $\times$ Trial Interaction: $F_{(14,154)}=1.24, P=0.25$; Fig. $\left.2 \mathrm{~A}\right)$. Additionally, there was no overall difference in shock reactivity between lesioned and nonlesioned animals $\left(t_{(11)}=0.16, P=0.88\right.$; Fig. 2B). Thus, lesions of the vmPFC did not appear to alter the experience of the traumatic event.

\section{Day 2-Trauma memory test}

When animals were placed back into Context A on the day after trauma, animals that received the traumatic stressor displayed high levels of freezing while nonshocked animals did not $(U=0$, $P<0.001$; Fig. 2C). However, there was no impact of lesion on freezing during this session (effect in trauma animals: $t_{(12)}=0.08$, $P=0.94$; effect in nontrauma animals: $t_{(13)}=0.94, P=0.36$ ). These findings suggest that lesions of the vmPFC did not alter the associative recall of the traumatic event.

A

Experiment 1

\begin{tabular}{|c|c|ccc|}
\multicolumn{1}{c}{ Day 1 } & Day 2 & Day 3 & Day 4 \\
\cline { 1 - 2 } & $\begin{array}{c}\text { Trauma } \\
\text { 0/15 Shocks } \\
\text { Context A }\end{array}$ & $\begin{array}{c}\text { Trauma Test } \\
\text { Context A }\end{array}$ & $\begin{array}{c}\text { Mild Stressor } \\
\text { 1 Startle Stimulus } \\
\text { Context B }\end{array}$ & $\begin{array}{c}\text { SEFL Context Test } \\
\text { Context B }\end{array}$ \\
\hline
\end{tabular}

B

\section{Experiment 2}
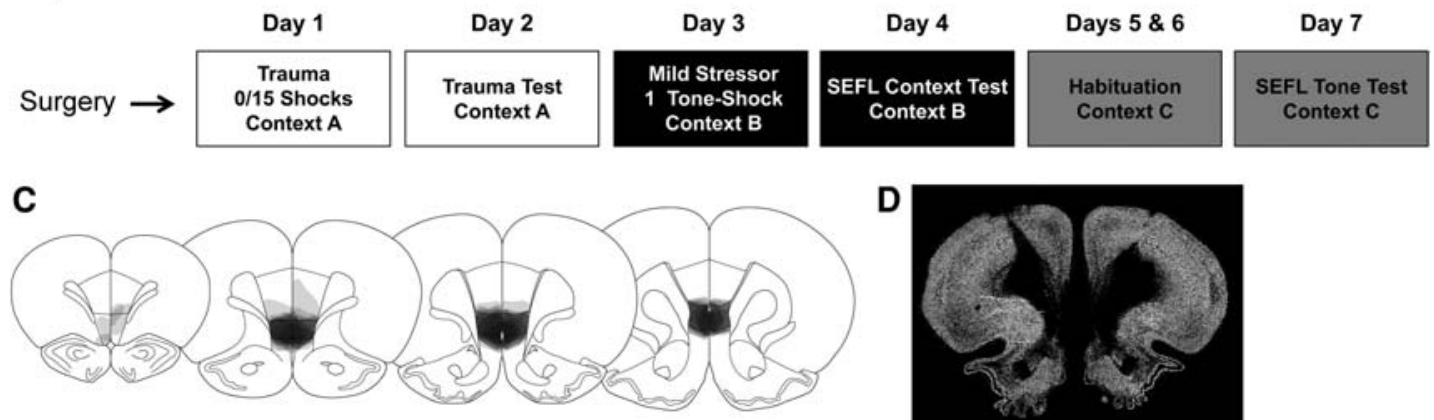

AP 4.2

AP 3.7

AP 3.0

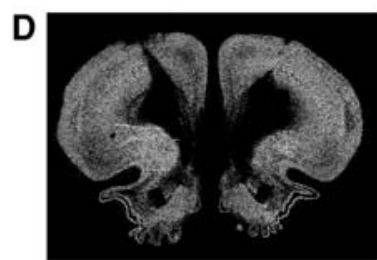

AP 2.5

Figure 1. Experimental timeline and depiction of lesion size. $(A, B)$ Experimental timeline for Experiment $1(A)$ and Experiment $2(B)$. Of note, $2 \mathrm{wk}$ were allowed for post-operative recovery. $(C)$ Distribution of lesion magnitude in the medial prefrontal cortex across animals in Experiment 1 . Each layer of shading represents a single animal. Coronal sections are labeled with respect to their distance anterior to bregma. Distribution of lesions was nearly identical in Experiment 2. (D) Representative immunofluorescent image of neuronal loss seen at the central focus of lesion by NeuN staining. 


\section{Experiment 1}

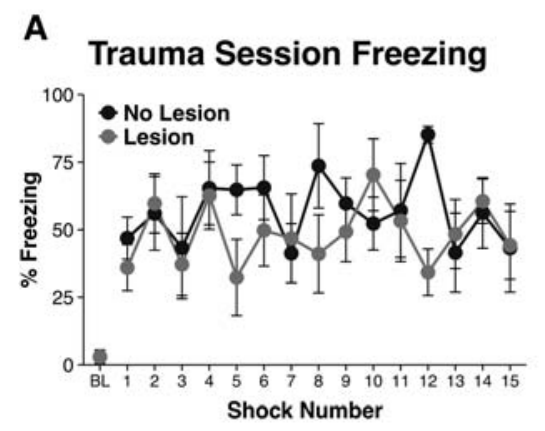

D

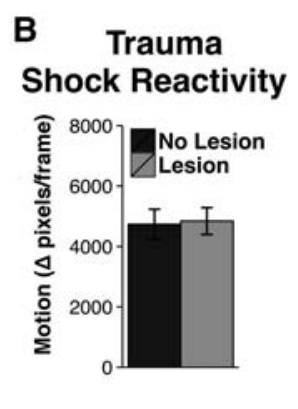

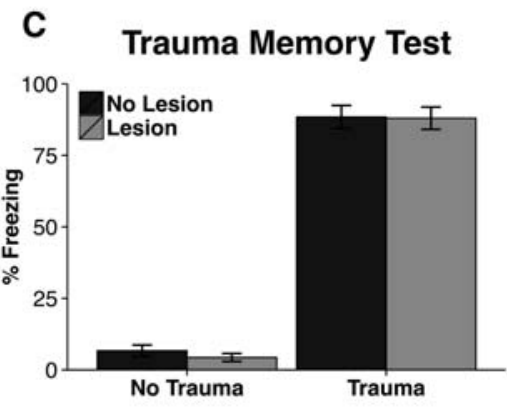

E
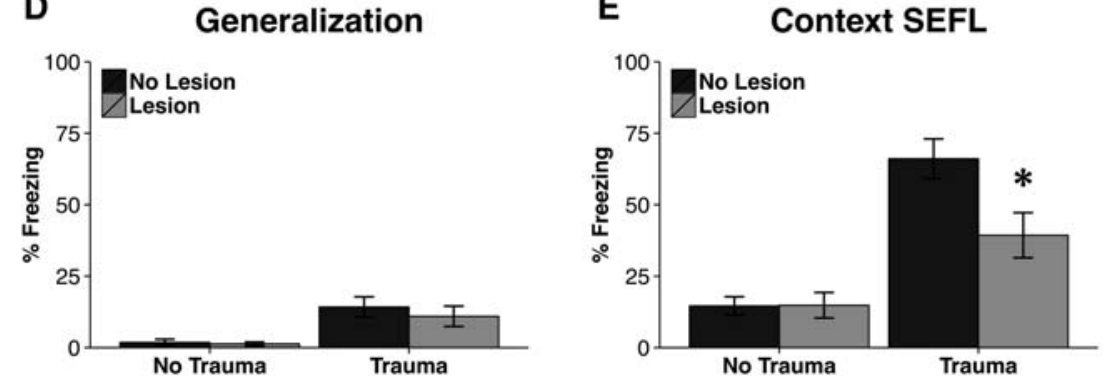

Figure 2. Results of Experiment 1. (A) Animals with lesions of the vmPFC did not differ in terms of baseline fear (BL) prior to the onset of the first shock nor did they show altered freezing throughout the trauma session. $(B)$ Lesion animals also did not differ in shock reactivity during the trauma session. (C) When placed back into the trauma context the subsequent day, lesion animals did not display altered fear. $(D)$ Fear of the traumatic context did not differentially generalize to a novel context between nonlesion and lesion animals, although animals that experienced the trauma did display significant generalization relative to no trauma controls. (E) After being exposed to a mild stressor in a novel context, animals that previously experienced the trauma showed increased freezing when placed back into the context associated with the mild stressor (evidence of SEFL). However, lesion animals showed an attenuation of this enhancement. Asterisk indicates significance at $P<0.05$. Error bars reflect standard error of the mean. "BL" $=$ Baseline.

\section{Day 3-Trauma generalization and mild stressor in a novel context}

All animals were placed in a novel context, Context $\mathrm{B}$, and were exposed to a single loud auditory stimulus ( $100 \mathrm{msec}, 115 \mathrm{~dB}$, white noise) after being allowed to explore the context for $3 \mathrm{~min}$. Although animals that had previously experienced the trauma generalized slightly to this context during this initial exploratory period, reflected in higher prestartle freezing levels relative to nonshocked animals $(\sim 12 \%$ freezing; $U=21, P<0.001)$, there was no effect of lesion on fear generalization (Fig. 2D; effect in trauma animals: $t_{(12)}=0.66, P=0.52$; nontrauma animals: $t_{(13)}=0.38, P=$ $0.71)$.

\section{Day 4-SEFL context test}

Upon returning to Context $\mathrm{B}$, animals that had received the trauma displayed higher levels of freezing in this startle paired-context than animals that did not receive trauma (Fig. $2 \mathrm{E} ; F_{(1,25)}=43.23, P$ $<0.001$ ), indicative of stress-enhanced fear learning (SEFL). However, lesioned animals displayed this enhancement to a lesser extent: an interaction between lesion and trauma was found $\left(F_{(1,25)}=5.47, P=0.03\right)$ and post hoc analysis revealed that lesioned animals in the trauma condition froze less than their nonlesion counterparts (Fig. 2E; $t_{(12)}=2.55, P=0.025$ ). There was no impact of lesion in nontraumatized animals on freezing in this test session $\left(t_{(13)}=0.963, P=0.96\right)$.

\section{Experiment 2: impact of vmPFC lesions on enhanced tone fear learning following trauma}

In order to assess whether the decrement in SEFL observed in Experiment 1 might be attributed to the fact that a contextual cue was used, Experiment 2 assessed whether enhanced fear learning for a discrete tone cue was similarly reduced in IL lesioned animals following trauma. An experimental timeline for Experiment 2 can be seen in Figure 1B.

\section{Histology}

The extent of lesion seen in Experiment 2 was identical to that seen in Experiment 1. Six of the animals that were infused with NMDA were excluded for misplaced or insufficient lesion.

\section{Day 1-Trauma}

As in Experiment 1, half of the animals received the 15-shock trauma on the first day of training in Context $\mathrm{A}$, whereas the other half of the animals was placed in Context A for an equivalent amount of time without shock. Lesions of the vmPFC again did not produce differences in freezing during the trauma session (Lesion: $F_{(1,23)}=$ 3.08, $P=0.09$; Lesion $\times$ Trial Interaction: $F_{(9,207)}=1.47, P=0.16$ ) nor did they impact shock reactivity $\left(t_{(23)}=0.94, P=0.36\right)$.

\section{Day 2-Trauma memory test}

As expected from the results of Experiment 1, traumatized animals froze more than nonshocked animals when returned to Context $\mathrm{A}$ $(U=4, P<0.001)$ and lesions of the vmPFC did not produce altered freezing in this test (effect in trauma animals: $t_{(23)}=0.77, P=0.45$; nontrauma animals: $\left.t_{(22)}=0.21, P=0.44\right)$. 


\section{Day 3-Trauma generalization and tone-shock pairing in novel context B}

In Experiment 2, animals were conditioned with a tone in a novel context in order to subsequently assess whether the reduction of fear observed in lesion animals in Experiment 1 was specific to contextual stimuli. Animals were placed into Context B and after a 3-min baseline period were presented with a single tone paired with shock. Although trauma animals froze to some extent in this novel context during the baseline period prior to the tone relative to nontrauma animals $(U=116, P>0.001)$, lesioned animals did not differ from controls (effect in trauma animals: $t_{(23)}=1.37$, $P=0.183$; effect in nontrauma animals: $\left.t_{(22)}=0.25, P=0.81\right)$, indicating that lesioned animals did not generalize any more than control animals.

\section{Day 4-SEFL context test}

Before proceeding to assess fear of the tone, animals were returned to Context B to assess fear of this context. In general, animals that received the trauma froze more during this test than animals that had not undergone tone-shock conditioning, evidence of SEFL $\left(F_{(1,45)}=7.05, P=0.01\right.$; Fig. $\left.3 \mathrm{~A}\right)$. However, in contrast to Experiment 1, there was no effect of lesion, nor was there a lesion $\times$ trauma interaction, on the level of freezing in this test of enhanced contextual fear (Lesion: $F_{(1,45)}=0, P=0.98$; Lesion $\times$ Trauma Interaction: $F_{(1,45)}=0.47, P=0.5$; Fig. $\left.3 \mathrm{~A}\right)$. Notably, freezing during this session in nonlesion trauma animals was significantly higher than in Experiment $1\left(t_{(19)}=2.12, P=0.05\right)$, possibly contributing to this discrepancy.

\section{Days 5-6-Habituation to novel context C}

So that fear of the tone could be assessed in the absence of any baseline contextual fear, tone fear was assessed in a novel context,

\section{A Experiment 2}
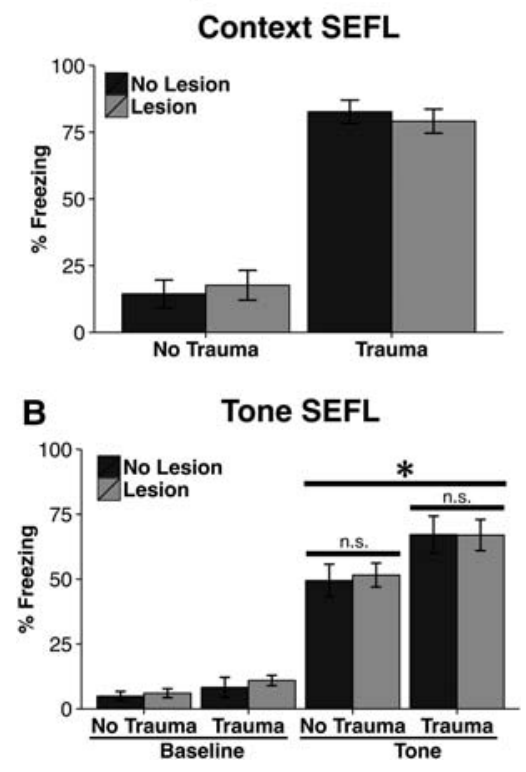

Figure 3. Results of Experiment 2. $(A)$ In Experiment 2, lesion and no lesion animals did not differ with respect to the enhancement of contextual fear learning seen following trauma, though freezing levels were generally higher in Experiment 2 relative to Experiment 1. (B) Animals that received the trauma showed enhanced fear of the tone paired with shock. Here, there was no difference in freezing between lesion and no lesion animals, irrespective of trauma condition. Asterisk indicates significance at $P<0.05$. Error bars reflect standard error of the mean.
Context C. In order to insure that there was no generalized fear to Context $\mathrm{C}$ when the tone was tested, animals were first habituated to novel Context $\mathrm{C}$ over the course of two days. The amount of generalized fear was assessed during the first $3 \mathrm{~min}$ of these habituation sessions. There was no effect of lesion $\left(F_{(1,45)}=0.144, P=\right.$ $0.71)$, lesion $\times$ trauma interaction $\left(F_{(1,45)}=0.1, P=0.76\right)$, or lesion $\times$ day $\times$ trauma interaction $\left(F_{(1,45)}=1.93, P=0.172\right)$, on the amount of freezing expressed.

\section{Day 7-SEFL tone test}

Prior to the tone being presented, all groups had equivalent levels of low baseline freezing $(P>0.1)$. Upon tone presentation, animals that experienced the trauma displayed significantly more fear of the tone than animals that had not, indicative of SEFL $\left(F_{(1,45)}=\right.$ 7.05, $P=0.01$; Fig. 3B). That being said, animals with lesions did not differ from their nonlesion counterparts (Lesion: $F_{(1,45)}=$ $0.02, P=0.88$; Lesion $\times$ Trauma Interaction: $F_{(1,45)}=0.04, P=0.85$; Fig. 3B). Thus, lesions of the vmPFC did not impact freezing to the tone in any way.

\section{Discussion}

The medial prefrontal cortex and its subregions are commonly placed within a framework of fear excitation and inhibition (Vidal-Gonzalez et al. 2006; Sierra-Mercado et al. 2011). This view has had a broad impact upon the field of psychiatry, with alterations in the function of the vmPFC being espoused to render individuals less able to inhibit fear responses generated within subcortical regions (Britton et al. 2005; Quirk and Beer 2006; Etkin and Wager 2007; Milad et al. 2009; Shvil et al. 2014; Do-Monte et al. 2015; Motzkin et al. 2015). Counter to this, the experiments above found that although vmPFC lesions had no impact on the acquisition or expression of a traumatic fear memory, and did not alter the ability of trauma to augment the subsequent acquisition of fear for a discrete tone paired with shock, it greatly diminished the ability of trauma to augment contextual fear learning. These findings point to a dramatically different, or perhaps additional, role of the vmPFC in fear regulation: contextual processing.

The idea that the vmPFC might contribute to contextual and/ or spatial processing is by no means new. It has long been known that among prefrontal subregions the vmPFC is the most richly interconnected with limbic structures that support contextual and/ or spatial processing (Hurley et al. 1991; Heidbreder and Groenewegen 2003; Uylings et al. 2003; Vertes 2004). Furthermore, Kesner et al. (1996) have highlighted the role of the vmPFC in working memory for spatial representations, but not stimuli in other sensory modalities (Ragozzino et al. 1998; Kesner and Ragozzino 2003). It is therefore not that surprising that these findings would extend to the realm of fear memories. Nevertheless, the contribution of the vmPFC to contextual/spatial processing has been poorly integrated with the contribution of the vmPFC to fear. This may be attributed to the lack of communication between neuroscience subfields, and furthermore, the predominant use of discrete cues (mostly tones) in studies of the vmPFC's contribution to fear (Santini et al. 2001, 2004; Sierra-Mercado et al. 2006, 2011; BurgosRobles et al. 2007; Do-Monte et al. 2015). Notably, similar to the absence of differences in freezing elicited by a shock-paired tone in vmPFC lesioned animals in Experiment 2, these studies have similarly failed to find differences in freezing to tone CSs.

It is important to highlight that the decrement in contextual fear conditioning seen in lesioned animals in Experiment 1 runs counter to other examples of contextual fear learning in these very experiments that were left intact. We speculate that these differences are a consequence of varying contextual processing demands emerging from the procedures used. 
With respect to the trauma memory tests, in which lesion animals and controls did not differ, animals were given a very long period of time to process the context $(90 \mathrm{~min})$ and displayed such high levels of fear that a deficit would be difficult to detect. Even in the case of the hippocampus, the bastion of spatial learning, pretraining lesions only produce deficits in contextual fear learning when animals are given a relatively short time to process the context, effectively increasing contextual demands (Wiltgen et al. 2006), or if the longevity of the memory is assessed long after learning (Zelikowsky et al. 2012).

Lesioned animals and controls also did not differ in Experiment 2 when we reexamined the enhancement of contextual fear learning. These differences may have emerged from varying contextual overlap as a result of the need to use three contexts in Experiment 2 as opposed to two in Experiment 1, or the use of a shock as the unconditional stimulus in Experiment 2 as opposed to an auditory startle stimulus in Experiment 1. Indeed, the impact of these procedural differences is evidenced in control animals, which displayed significantly higher levels of fear in the test of enhanced contextual fear in Experiment 2 relative to Experiment 1. Therefore, failure to find a decrement in lesioned animals in Experiment 2 may represent a ceiling effect. However, given that our laboratory has previously found contextual fear learning differences in vmPFC lesioned animals when shock was used as the unconditional stimulus (Zelikowsky et al. 2013b), and other labs have provided similar results with mPFC manipulations when using shocks (Antoniadis and McDonald 2006; Xu and Südhof 2013), it is unlikely that the differences observed result from a specific deficit in processing the startle stimulus. Simple contextual fear conditioning using shock also alters the excitability of IL neurons (Soler-Cedeño et al. 2016). Rather, the intensity of these two unconditional stimuli might support differential amounts of contextual fear learning, which in turn altered the impact of vmPFC lesions.

In any event, we do not intend to make the claim that the contributions of the vmPFC to contextual fear learning are anything but nuanced. In a previous report from our laboratory, lesions of the vmPFC only modestly reduced contextual fear, but simultaneously increased contextual fear generalization when animals were placed in a novel context. However, full disruptions of contextual fear were seen in animals that also had lesions of the hippocampus, or when the hippocampus and the vmPFC were disconnected (Zelikowsky et al. 2013b). Such results suggest that the vmPFC supports the fidelity of contextual fear memories, often in concert with other brain regions that are able to compensate for one another. The current findings add to the varied list of situations in which the vmPFC contributes to contextual processing in fear learning. In accordance with findings that the vmPFC contributes to spatial working memory in food-motivated tasks (Kesner et al. 1996; Ragozzino et al. 1998; Kesner and Ragozzino 2003), it is possible that the vmPFC contributes to contextual processing during fear learning when working memory demand is high: for instance, when a context is novel and its features must be integrated rapidly. Future work ought to disentangle the precise circumstances under which the vmPFC contributes to contextual fear learning.

It is interesting that at no time during experimental testing did animals with lesions of the vmPFC display evidence of heightened fear responses. This runs in stark contrast to several reports that activation of the vmPFC is able to reduce anxiety-like behaviors, whereas inhibition produces the opposite response (SierraMercado et al. 2011; Adhikari et al. 2015; Do-Monte et al. 2015). Nevertheless, these findings are also consistent with several reports that inactivation of the vmPFC or its infralimbic subregion do not impact expression of fear during fear acquisition (Sierra-Mercado et al. 2006, 2011; Zelikowsky et al. 2013b). A previous study from our own laboratory similarly found that lesions of the vmPFC were without effect on anxiety behaviors in the elevated plus maze (Zelikowsky et al. 2013b). Therefore, a simple excitation/inhibition view of the vmPFC in the regulation of fear must be reconsidered. Perhaps, such impacts on fear expression are a byproduct of an organism being uncertain of the context, as context plays an important role in regulating the expression of fear behaviors, particularly following extinction (Bouton 1984).

In closing, contextual processing plays a pivotal role in allowing organisms to constrain fear to appropriate settings, including the modulation of extinction memories. Future studies targeting the precise means through which the vmPFC supports contextual processing might therefore shed light on how disruption of this brain region in PTSD patients contributes to symptomology.

\section{Materials and Methods}

\section{Animals}

A total of 87 male, Long Evans rats, weighing between 300-350 g at the time of surgery, were used for the present experiments (Experiment 1: $n=32$; Experiment 2: $n=55$ ). Rats had food and water available ad libitum, except when taken out of the vivarium for testing, and were housed on a 12-h on/off light cycle. All animals were housed in isolation for $1 \mathrm{wk}$ prior to beginning behavioral testing. During this time they were handled for $\sim 1$ min per day. The Chancellor's Animal Research Committee at UCLA approved all procedures involving animals.

\section{Surgery}

Surgical procedures followed standard aseptic technique. Following induction of anesthesia with isoflurane and administration of the anti-inflammatory carprofen $(5 \mathrm{mg} / \mathrm{kg}$, s.c.), the skull was exposed and small holes were drilled on either side. Injectors, connected to a syringe pump via polyethylene tubing, were lowered to the following millimeter coordinates relative to bregma at a 30 degree angle: $\mathrm{AP}=2.5, \mathrm{ML}=0.4, \mathrm{DV}=-5.5$. A volume of $0.1 \mu \mathrm{L}$ of NMDA $(20 \mathrm{mg} / \mathrm{mL}$, dissolved in $1 \times$ PBS) or an equivalent volume of vehicle was infused at a rate $0.1 \mu \mathrm{L} / \mathrm{min}$. Injectors were left in place for $10 \mathrm{~min}$ prior to being removed from the brain. The scalp wound was then flushed with saline prior to being closed with wound-clips. Animals were given an additional injection of carprofen $24 \mathrm{~h}$ after surgery and were maintained on an antibiotic solution of trimethoprim and sulfamethoxazole for $5 \mathrm{~d}$ following surgery. Animals were given 2 wk to recover prior to behavioral training/testing.

\section{Behavioral testing}

\section{Apparatus and data acquisition}

All behavioral testing took place in Med Associates (Med Associates) conditioning chambers, controlled via Med Associates Video Freeze software, as described previously (Zelikowsky et al. 2013a,b). Chambers were configured to represent distinct contexts, differing in physical appearance, luminosity, odor, and background noise. Notably, although each context (A, B, and C) was the same for all animals within an experiment, across experiments their features varied. Transport boxes to the different contexts were also varied to aid in discriminability. Scrambled shocks were delivered to grid floors in the chambers via Med Associates shock scramblers (ENV 414-S) and auditory startle stimuli were delivered using Goldwood GT-1005 wide dispersion piezo tweeters mounted to the wall of the chambers and connected to an amplifier. Pure tones were delivered using Med Associates cage speakers (ENV-224AM). Sessions were recorded by near infrared cameras and freezing and motion were measured using Med Associates Video Freeze software. Using this software, motion was calculated as the average number of pixels whose gray scale value changed per frame (30 frames/sec) during the specified time. Freezing was defined as motion below a threshold that conformed to visual 
inspection of behavior (Zelikowsky et al. 2012; 2013a,b; Poulos et al. 2014, 2015; Perusini et al. 2016).

\section{Experiment 1}

Day 1-Trauma. On the first day of behavioral testing, animals were transported in cages from the vivarium and placed in Context A, the trauma context, for a total of $90 \mathrm{~min}$. Over this time period, animals in the trauma condition received $15,1 \mathrm{sec}$, $1 \mathrm{~mA}$ unsignaled footshocks on a pseudorandom schedule. Nonshocked animals remained in Context A for an equivalent amount of time. Motion was recorded during the time of shock to measure shock reactivity, and the percentage of time spent freezing was scored during a $30 \mathrm{sec}$ period beginning $30 \mathrm{sec}$ after the termination of each shock.

Day 2-Trauma memory test. Animals were placed back into Context A for $8 \mathrm{~min}$ and freezing was assessed.

Day 3-Trauma generalization and mild stressor in a novel context. After a 3 min baseline period in Context B, all animals experienced a $100 \mathrm{msec}$, $115 \mathrm{~dB}, 0$ msec rise time, white noise, startle stimulus. They were removed from the context $30 \mathrm{sec}$ later. Freezing was examined during the first $3 \mathrm{~min}$, as this provides a metric of generalized fear.

Day 4-SEFL context test. Animals were placed back into Context B for 8 min and freezing was assessed.

\section{Experiment 2}

Day 1 - Trauma. Day 1 for Experiment 2 was identical to Experiment 1 , in which animals received zero or 15 shocks in Context A.

Day 2-Trauma test. Animals were placed back into Context A for 8 min and freezing was assessed.

Day 3-Trauma generalization and mild stressor in a novel context. After a 3 min baseline period in Context $\mathrm{B}$, all animals experienced a $30 \mathrm{sec}, 75$ $\mathrm{dB}, 2800 \mathrm{~Hz}$ pure tone, coterminating with a $1 \mathrm{sec}$, one $\mathrm{mA}$, footshock. They were removed from the Context $30 \mathrm{sec}$ later. Freezing was examined during the first $3 \mathrm{~min}$ to assess fear generalization.

Day 4-SEFL context test. Animals were placed back into Context B on Day 4 for $8 \mathrm{~min}$ and freezing was assessed. This was done in order to assess contextual fear of the tone-shock context, for which a fear memory is also formed, and for which traumatized animals show increased contextual fear (i.e., SEFL).

Days 5-6-Habituation to novel context $C$. In order so that fear of the tone could be assessed in the absence of any baseline contextual fear, animals' fear of the tone was assessed in a novel context, Context C. Because animals showed some generalization of fear to this context, they were first habituated to Context $\mathrm{C}$ during two half hour long sessions. Freezing was examined during the first $3 \mathrm{~min}$ of these sessions.

Day 7. SEFL tone test. After a $3 \mathrm{~min}$ baseline during which baseline freezing was assessed, animals were presented with the tone used during conditioning on Day 3 a total of four times, each separated by $90 \mathrm{sec}$. Freezing was averaged across the tone periods after initial inspection of the data revealed no effects of trial number.

\section{Histology}

Brains were rapidly extracted and post-fixed in $4 \%$ paraformaldehyde solution at $4^{\circ} \mathrm{C}$ overnight before being switched to a $30 \%$ sucrose solution and allowed to sink over the course of a few days. Brains were then frozen and sectioned at 40 microns using a cryostat. Every fifth section was collected in wells of $1 \times$ PBS for staining, starting near the rostral pole of the prefrontal cortex and proceeding until the vmPFC was no longer visible. Tissue was stained for the neuron-specific marker NeuN. The protocol for NeuN staining is as follows. Tissue was first blocked for $1 \mathrm{~h}$ (5\% normal goat serum, $0.5 \%$ Triton X-100, in PBS) and was then incubated in primary antibody overnight at $4^{\circ} \mathrm{C}$ (EMD Millipore Rabbit Polyclonal Anti-NeuN, \# ABN78; diluted to 1:2000 in blocking solution). The next morning, the tissue was washed using PBS before being incubated in a fluorescent secondary antibody for $2 \mathrm{~h}$ at room temperature (Invitrogen Goat Anti-Rabbit IgG, Alexa Fluor 594, \# A-11037). After a final set of washes, tissue was mounted onto slides and coverslipped using Vectashield containing DAPI (Vector Labs). Tissue was visually examined using a Keyence BZ-X710 fluorescent microscope and spread of the lesion, indicated by total a loss of NeuN staining, was traced onto coronal sections from the rat brain atlas of Paxinos and Watson (2007) using GIMP software (http://www. gimp.org/).

\section{Acknowledgments}

This study was funded by grants from RO1MH062122 to M.S.F. and F31MH185207-02 to Z.T.P.

\section{References}

Adhikari A, Lerner TN, Finkelstein J, Pak S, Jennings JH, Davidson TJ, Ferenczi E, Gunaydin LA, Mirzabekov JJ, Ye L, et al. 2015. Basomedial amygdala mediates top-down control of anxiety and fear. Nature 527: 179-185.

Amat J, Baratta MV, Paul E, Bland ST, Watkins LR, Maier SF. 2005. Medial prefrontal cortex determines how stressor controllability affects behavior and dorsal raphe nucleus. Nat Neurosci 8: 365-371.

Antoniadis EA, McDonald RJ. 2006. Fornix, medial prefrontal cortex, nucleus accumbens, and mediodorsal thalamic nucleus: roles in a fear-based context discrimination task. Neurobiol Learn Mem 85: 71-85.

Bouton ME. 1984. Differential control by context in the inflation and reinstatement paradigms. J Exp Psychol Anim Behav Process 10: 56-74.

Britton JC, Phan KL, Taylor SF, Fig LM, Liberzon I. 2005. Corticolimbic blood flow in posttraumatic stress disorder during script-driven imagery. Biol Psychiatry 57: 832-840.

Burgos-Robles A, Vidal-Gonzalez I, Santini E, Quirk GJ. 2007. Consolidation of fear extinction requires NMDA receptor-dependent bursting in the ventromedial prefrontal cortex. Neuron 53: 871-880.

Do-Monte FH, Manzano-Nieves G, Quiñones-Laracuente K, Ramos-Medina L, Quirk GJ. 2015. Revisiting the role of infralimbic cortex in fear extinction with optogenetics. J Neurosci 35: 3607-3615.

Etkin A, Wager TD. 2007. Functional neuroimaging of anxiety: a meta-analysis of emotional processing in PTSD, social anxiety disorder, and specific phobia. Am J Psychiatry 164: 1476-1488.

Heidbreder CA, Groenewegen HJ. 2003. The medial prefrontal cortex in the rat: evidence for a dorso-ventral distinction based upon functional and anatomical characteristics. Neurosci Biobehav Rev 27: 555-579.

Hurley KM, Herbert H, Moga MM, Saper CB. 1991. Efferent projections of the infralimbic cortex of the rat. J Comp Neurol 308: 249-276.

Kasai K, Yamasue H, Gilbertson MW, Shenton ME, Rauch SL, Pitman RK. 2008. Evidence for acquired pregenual anterior cingulate gray matter loss from a twin study of combat-related posttraumatic stress disorder. Biol Psychiatry 63: 550-556.

Kesner RP, Ragozzino ME. 2003. The role of the prefrontal cortex in object-place learning: a test of the attribute specificity model. Behav Brain Res 146: 159-165.

Kesner RP, Hunt ME, Williams JM, Long JM. 1996. Prefrontal cortex and working memory for spatial response, spatial location, and visual object information in the rat. Cereb Cortex 6: 311-318.

Koenigs M, Grafman J. 2009. Posttraumatic stress disorder: the role of medial prefrontal cortex and amygdala. Neuroscientist 15: 540-548.

Kühn S, Gallinat J. 2013. Gray matter correlates of posttraumatic stress disorder: a quantitative meta-analysis. Biol Psychiatry 73: 70-74.

Laurent V, Westbrook RF. 2009. Inactivation of the infralimbic but not the prelimbic cortex impairs consolidation and retrieval of fear extinction. Learn Mem 16: 520-529.

Milad MR, Quinn BT, Pitman RK, Orr SP, Fischl B, Rauch SL. 2005. Thickness of ventromedial prefrontal cortex in humans is correlated with extinction memory. Proc Natl Acad Sci 102: 10706-10711.

Milad MR, Pitman RK, Ellis CB, Gold AL, Shin LM, Lasko NB, Zeidan MA, Handwerger K, Orr SP, Rauch SL. 2009. Neurobiological basis of failure to 
recall extinction memory in posttraumatic stress disorder. Biol Psychiatry 66: $1075-1082$.

Motzkin JC, Philippi CL, Wolf RC, Baskaya MK, Koenigs M. 2015. Ventromedial prefrontal cortex is critical for the regulation of amygdala activity in humans. Biol Psychiatry 77: 276-284.

Paxinos G, Watson C. 2007. The rat brain in stereotaxic coordinates, 6 th ed. Academic Press, Cambridge, MA.

Perusini JN, Meyer EM, Long VA, Rau V, Nocera N, Avershal J, Maksymetz J, Spigelman I, Fanselow MS. 2016. Induction and expression of fear sensitization caused by acute traumatic stress. Neuropsychopharmacology 41: $45-57$.

Phelps EA, Delgado MR, Nearing KI, LeDoux JE. 2004. Extinction learning in humans: role of the amygdala and vmPFC. Neuron 43: 897-905.

Poulos AM, Reger M, Mehta N, Zhuravka I, Sterlace SS, Gannam C, Hovda DA, Giza CC, Fanselow MS. 2014. Amnesia for early life stress does not preclude the adult development of posttraumatic stress disorder symptoms in rats. Biol Psychiatry 76: 306-314.

Poulos AM, Zhuravka I, Long V, Gannam C, Fanselow M. 2015. Sensitization of fear learning to mild unconditional stimuli in male and female rats. Behav Neurosci 129: 62-67.

Quirk GJ, Beer JS. 2006. Prefrontal involvement in the regulation of emotion: convergence of rat and human studies. Curr Opin Neurobiol 16: 723-727.

Ragozzino ME, Adams S, Kesner RP. 1998. Differential involvement of the dorsal anterior cingulate and prelimbic-infralimbic areas of the rodent prefrontal cortex in spatial working memory. Behav Neurosci 112: 293-303.

Rau V, Fanselow MS. 2009. Exposure to a stressor produces a long lasting enhancement of fear learning in rats. Stress 12: 125-133.

Rau V, DeCola JP, Fanselow MS. 2005. Stress-induced enhancement of fear learning: an animal model of posttraumatic stress disorder. Neurosci Biobehav Rev 29: 1207-1223.

Rauch SL, Shin LM, Segal E, Pitman RK, Carson MA, McMullin K, Whalen PJ, Makris N. 2003. Selectively reduced regional cortical volumes in post-traumatic stress disorder. Neuroreport 14: 913-916.

Richert KA, Carrion VG, Karchemskiy A, Reiss AL. 2006. Regional differences of the prefrontal cortex in pediatric PTSD: an MRI study. Depress Anxiety 23: $17-25$.

Santini E, Muller RU, Quirk GJ. 2001. Consolidation of extinction learning involves transfer from NMDA-independent to NMDA-dependent memory. J Neurosci 21: 9009-9017.

Santini E, Ge H, Ren K, Peña de Ortiz S, Quirk GJ. 2004. Consolidation of fear extinction requires protein synthesis in the medial prefrontal cortex. $J$ Neurosci 24: 5704-5710.
Shvil E, Sullivan GM, Schafer S, Markowitz JC, Campeas M, Wager TD, Milad MR, Neria Y. 2014. Sex differences in extinction recall in posttraumatic stress disorder: a pilot fMRI study. Neurobiol Learn Mem 113: $101-108$.

Sierra-Mercado D Jr, Corcoran KA, Lebrón-Milad K, Quirk GJ. 2006. Inactivation of the ventromedial prefrontal cortex reduces expression of conditioned fear and impairs subsequent recall of extinction. Eur $J$ Neurosci 24: 1751-1758.

Sierra-Mercado D, Padilla-Coreano N, Quirk GJ. 2011. Dissociable roles of prelimbic and infralimbic cortices, ventral hippocampus, and basolateral amygdala in the expression and extinction of conditioned fear. Neuropsychopharmacology 36: 529-538.

Soler-Cedeño O, Cruz E, Criado-Marrero M, Porter JT. 2016. Contextual fear conditioning depresses infralimbic excitability. Neurobiol Learn Mem 130: $77-82$.

Uylings HB, Groenewegen HJ, Kolb B. 2003. Do rats have a prefrontal cortex? Behav Brain Res 146: 3-17.

Vertes RP. 2004. Differential projections of the infralimbic and prelimbic cortex in the rat. Synapse 51: 32-58.

Vidal-Gonzalez I, Vidal-Gonzalez B, Rauch SL, Quirk GJ. 2006. Microstimulation reveals opposing influences of prelimbic and infralimbic cortex on the expression of conditioned fear. Learn Mem 13: 728-733.

Wiltgen BJ, Sanders MJ, Anagnostaras SG, Sage JR, Fanselow MS. 2006 Context fear learning in the absence of the hippocampus. J Neurosci 26: 5484-5491.

Xu W, Südhof TC. 2013. A neural circuit for memory specificity and generalization. Science 339: 1290-1295.

Zelikowsky M, Bissiere S, Fanselow MS. 2012. Contextual fear memories formed in the absence of the dorsal hippocampus decay across time. $J$ Neurosci 32: 3393-3397.

Zelikowsky M, Hast TA, Bennett RZ, Merjanian M, Nocera NA, Ponnusamy R, Fanselow MS. 2013a. Cholinergic blockade frees fear extinction from its contextual dependency. Biol Psychiatry 73: 345-352.

Zelikowsky M, Bissiere S, Hast TA, Bennett RZ, Abdipranoto A, Vissel B, Fanselow MS. 2013b. Prefrontal microcircuit underlies contextual learning after hippocampal loss. Proc Natl Acad Sci 110: 9938-9943.

Received June 20, 2017; accepted in revised form July 8, 2017. 


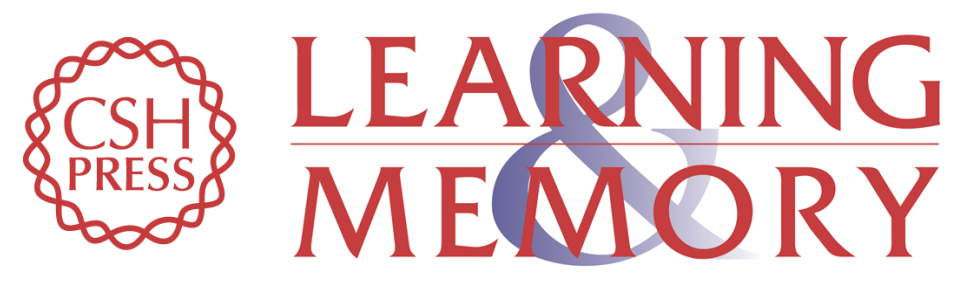

\title{
The ventromedial prefrontal cortex in a model of traumatic stress: fear inhibition or contextual processing?
}

\author{
Zachary T. Pennington, Austin S. Anderson and Michael S. Fanselow
}

Learn. Mem. 2017, 24:

Access the most recent version at doi:10.1101/Im.046110.117

\begin{aligned} & \hline References $\begin{array}{l}\text { This article cites } 43 \text { articles, } 10 \text { of which can be accessed free at: } \\ \text { http://learnmem.cshlp.org/content/24/9/400.full.html\#ref-list-1 }\end{array} \\ & \begin{array}{r}\text { Creative } \\ \text { Commons } \\ \text { License }\end{array} \begin{array}{l}\text { This article is distributed exclusively by Cold Spring Harbor Laboratory Press for the } \\ \text { first } 12 \text { months after the full-issue publication date (see } \\ \text { http://learnmem.cshlp.org/site/misc/terms.xhtml). After } 12 \text { months, it is available under } \\ \text { a Creative Commons License (Attribution-NonCommercial } 4.0 \text { International), as } \\ \text { described at http://creativecommons.org/licenses/by-nc/4.0/. }\end{array} \\ & \begin{array}{c}\text { Receive free email alerts when new articles cite this article - sign up in the box at the } \\ \text { top right corner of the article or click here. }\end{array} \\ & \begin{array}{l}\text { Service } \\ \text { terting }\end{array}\end{aligned}$

